\title{
Primary Neurolymphomatosis of the Lower Cranial Nerves Presenting as Dysphagia and Hoarseness: A Case Report
}

\author{
Naoto Sakai ${ }^{1}$ Tae Ito-Yamashita ${ }^{1}$ Goro Takahashi ${ }^{2}$ Satoshi Baba ${ }^{3}$ Shinichiro Koizumi ${ }^{1}$ \\ Tomohiro Yamasaki ${ }^{1}$ Tsutomu Tokuyama ${ }^{1}$ Hiroki Namba ${ }^{1}$
}

${ }^{1}$ Department of Neurosurgery, Hamamatsu University School of Medicine, Handayama, Higashiku, Hamamatsu, Japan

2 Department of Otolaryngology, Hamamatsu University School of Medicine, Handayama, Higashiku, Hamamatsu, Japan

${ }^{3}$ Department of Diagnostic Pathology, Hamamatsu University School of Medicine, Handayama, Higashiku, Hamamatsu, Japan

J Neurol Surg Rep 2014;75:e62-e66.
Address for correspondence Naoto Sakai, MD, PhD, Department of Neurosurgery, Hamamatsu University School of Medicine, Handayama 1-20-1, Higashiku, Hamamatsu 431-3192, Japan (e-mail: nsakaineurosurg@gmail.com).

\begin{abstract}
Keywords

- cranial nerve

- large B-cell lymphoma

- lower cranial nerves

- malignant lymphoma

- neurolymphomatosis

Primary neurolymphomatosis is an extremely rare tumor. We report the case of a 74-year-old patient presenting with dysphagia and hoarseness. Initial contrast-enhanced computed tomography of the head, neck, and chest did not reveal any lesions. His symptoms improved with short-term administration of prednisone but recurred and deteriorated. Magnetic resonance (MR) imaging revealed a tumor along the ninth and tenth cranial nerves across the jugular foramen. Fluorine-18 fluorodeoxyglucose positron emission tomography indicated this was a primary tumor. Repeated MR imaging after 2 months revealed considerable tumor enlargement. A left suboccipital craniotomy was performed to remove the tumor that infiltrated the ninth and tenth cranial nerves. The histopathologic diagnosis was diffuse large B-cell lymphoma. Although focal radiation therapy was administered to ensure complete eradication of the tumor, the patient died of aspiration pneumonia with systemic metastasis. To our knowledge, this is the first reported case of primary neurolymphomatosis in the lower cranial nerves.
\end{abstract}

\section{Introduction}

Neurolymphomatosis was first described in 2003 as a lymphoma entity that affects cranial and peripheral nerves and roots. ${ }^{1}$ Until that report, lymphoma infiltration in the roots and nerves had not been clearly differentiated from paraneoplastic or therapy-related root and nerve disorders. A primary neurolymphomatosis is defined as the first manifestation of the hematologic malignancy, whereas a secondary neurolymphomatosis is defined as a site of relapse or the progression of a previously diagnosed lymphoma or leukemia. ${ }^{2}$ In primary neurolymphomatosis, tumor infiltration into the affected neural structure has to be established by a biopsy or upon autopsy. ${ }^{2}$ Several studies have reported secondary neurolymphomatosis of the cranial nerves. ${ }^{3-5}$ However, diagnosis of primary neurolymphomatosis of the cranial nerves remains difficult, and the difference between primary neurolymphomatosis of the cranial nerves and primary central nervous system lymphoma (PCNSL) has not been well documented. To the best of our knowledge, only two case reports of primary neurolymphomatosis of the cranial nerves have been published. ${ }^{6,7}$ We report a case of primary neurolymphomatosis of the lower cranial nerves presenting as dysphasia and vocal cord palsy that was confirmed pathologically as received

December 29, 2012

accepted after revision

November 3, 2013

published online

January 16, 2014
DOI http://dx.doi.org/ 10.1055/s-0033-1363505. ISSN 2193-6358. (c) 2014 Georg Thieme Verlag KG, Stuttgart · New York
License terms

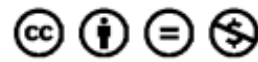




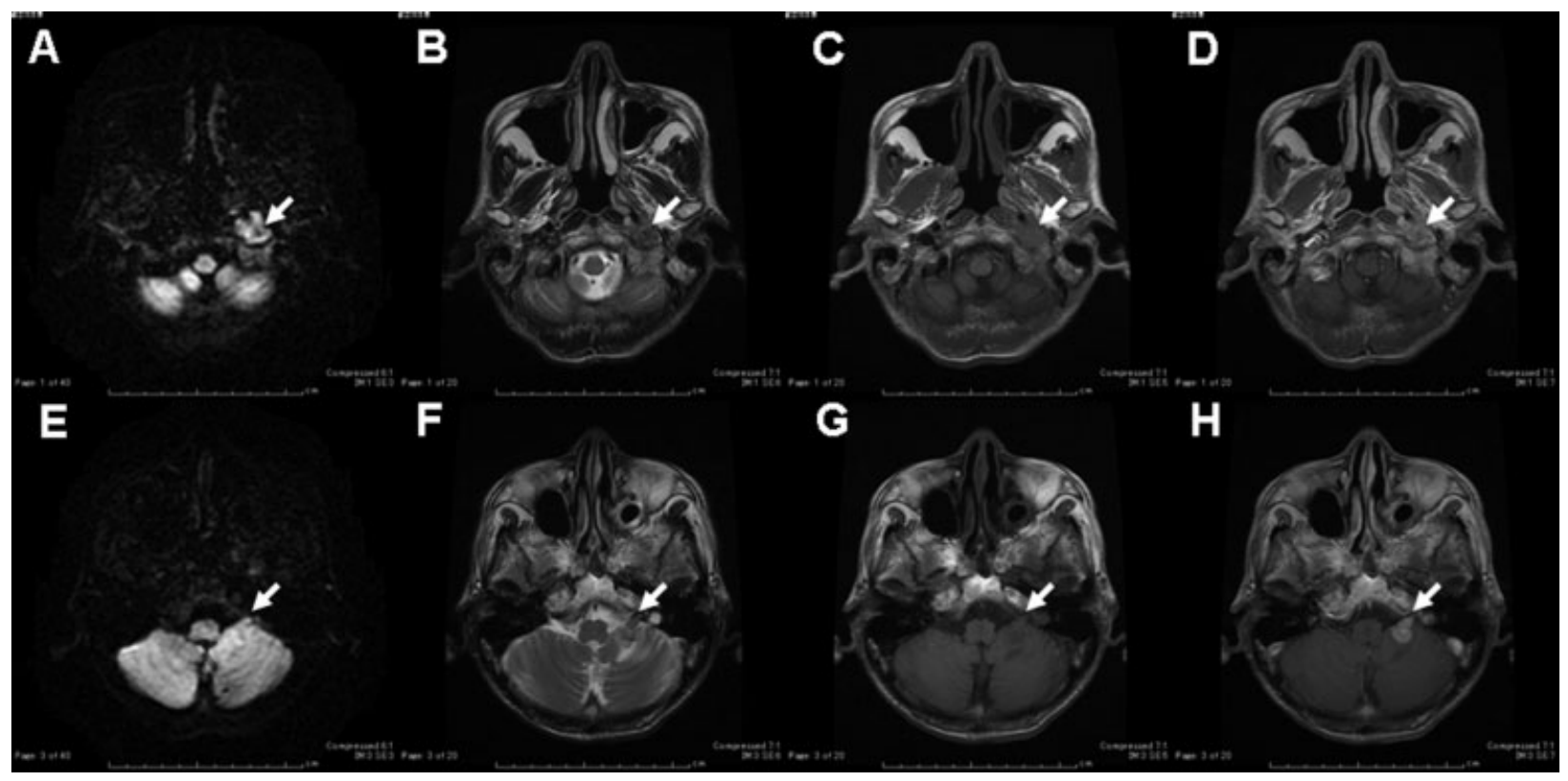

Fig. 1 Magnetic resonance imaging of a mass lesion between the parapharyngeal space and cerebellomedullary angle across the jugular foramen. The lesion was isointense to hyperintense on diffusion-weighted images (A, E), isointense to hyperintense on T2-weighted images (B, F), isointense to hypointense on T1-weighted images (C, G), and homogeneously enhanced with gadolinium (D, H).

diffuse large B-cell lymphoma. This is the first reported case of primary neurolymphomatosis of the lower cranial nerves.

\section{Case Report}

A 74-year-old man with a history of gastric cancer developed progressive dysphagia and hoarseness caused by left glossopharyngeal and vagus nerve palsies in July 2008. He was referred to the Otolaryngology Department at our university hospital from a private clinic. Contrast-enhanced head, neck, and chest computed tomography (CT) did not reveal any lesions. Gastric endoscopy did not show any recurrence of gastric cancer. He was referred to the Department of Neurology, where he was diagnosed with nonneoplastic cranial neuropathies. He was treated for 16 days with prednisone (20 mg/day). His dysphasia and vocal cord palsy improved temporarily but recurred and worsened in February 2009. Again, gastric endoscopy and chest and abdominal CT did not reveal any lesions.

In August 2009, gadolinium-enhanced brain magnetic resonance (MR) imaging demonstrated a mass lesion between the parapharyngeal space and cerebellomedullary angle along the ninth and tenth cranial nerves across the left jugular foramen ( $\boldsymbol{- F i g . ~ 1 ) . ~ T h e ~ l e s i o n ~ w a s ~ i s o i n t e n s e ~ t o ~}$ hypointense on T1-weighted imaging and isointense to hyperintense on diffusion-weighted imaging (DWI) and T2weighted imaging. It was homogeneously enhanced with gadolinium. We performed fluorine-18 fluorodeoxyglucose (FDG) positron emission tomography (PET) CT in September 2009 that detected the parapharyngeal space and cerebellomedullary angle mass lesions, but no other systemic lesions were detected. His serum soluble interleukin-2 receptor level $(1120 \mathrm{U} / \mathrm{mL})$ was higher than the normal range
(145-519 U/mL). Based on the MR imaging results and the high level of soluble interleukin-2 receptor, a primary malignant lymphoma of the lower cranial nerves was suspected. He was subsequently referred to the Hematology Division in the Department of Internal Medicine. However, the possibility of malignant lymphoma was considered low because FDG PETCT did not show any systemic lesions.

He was then referred to the Department of Neurosurgery at Hamamatsu University Hospital to obtain a biopsy sample from the intracranial lesion, but he refused craniotomy. After 2 months, he developed dysphagia, left occipital head and neck pain, gait disturbance, and general fatigue. He was admitted to our hospital in October 2009. Upon admission, he presented with palsies in the left eleventh and twelfth cranial nerves, in addition to the ninth and tenth cranial nerves. MR imaging revealed considerable tumor enlargement both in the intracranial and parapharyngeal space (-Fig. 2). The tumor was treated with methylprednisolone, and a tracheotomy was performed to prevent aspiration pneumonia.

Following the diagnosis of a primary lower cranial nerve malignant tumor, the lesion was explored by performing a left lateral suboccipital craniotomy in November 2009 (-Fig. 3). During surgery, an extra-arachnoid tumor involving the ninth and tenth cranial nerves was observed. There was no clear plane between the tumor and nerves; therefore, an infiltrating lesion was suspected. Partial resection of the tumor was performed following the intraoperative diagnosis of a malignant tumor. The seventh and eighth cranial nerves were not affected by tumor infiltration.

A histopathologic diagnosis of diffuse large B-cell lymphoma was made. Immunohistochemical staining demonstrated that the tumor was positive for cluster of differentiation 20 

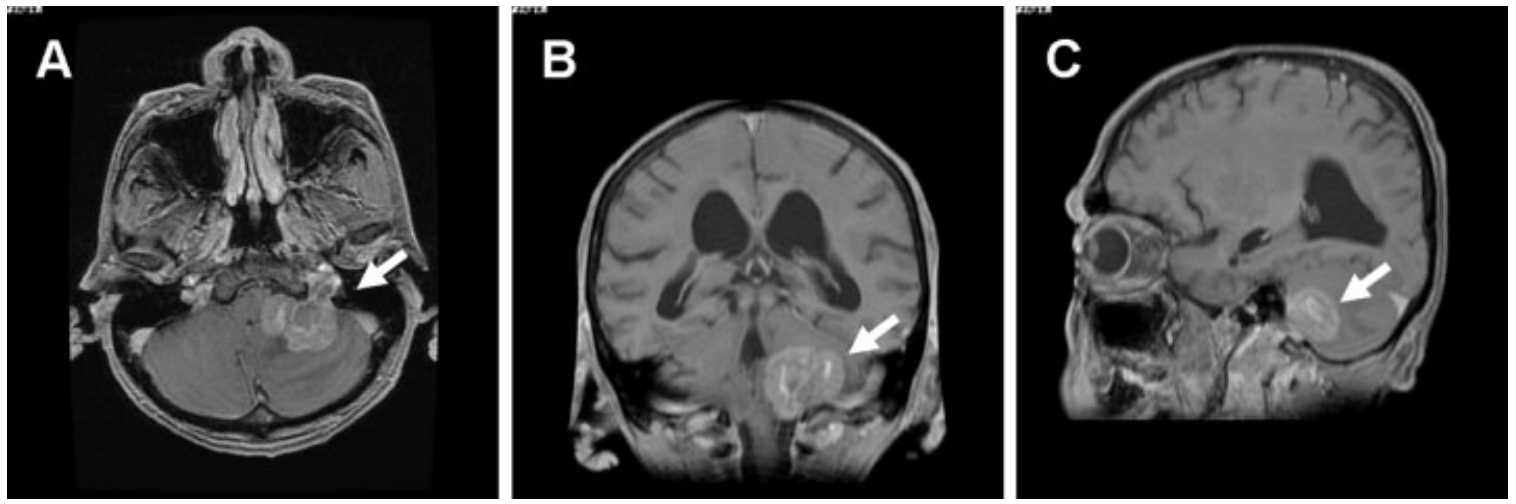

Fig. 2 (A-C) Gadolinium-enhanced T1-weighted magnetic resonance (MR) imaging. The image was obtained 3 months after the initial MR imaging (Fig. 1) and shows a considerable tumor enlargement both in the intracranial and parapharyngeal spaces.

(CD20), CD79a, B-cell lymphoma 6, and multiple myeloma oncogene 1 but negative for CD30, CD10, CD3, and CD5 (-Fig. 4). The tumor was also negative for Epstein-Barr virus-encoded small RNAs, as detected by in situ hybridization.

Because of the patient's poor condition, local radiotherapy, but not chemotherapy, was initiated to relieve the occipital and neck pain. MR imaging demonstrated complete remis-
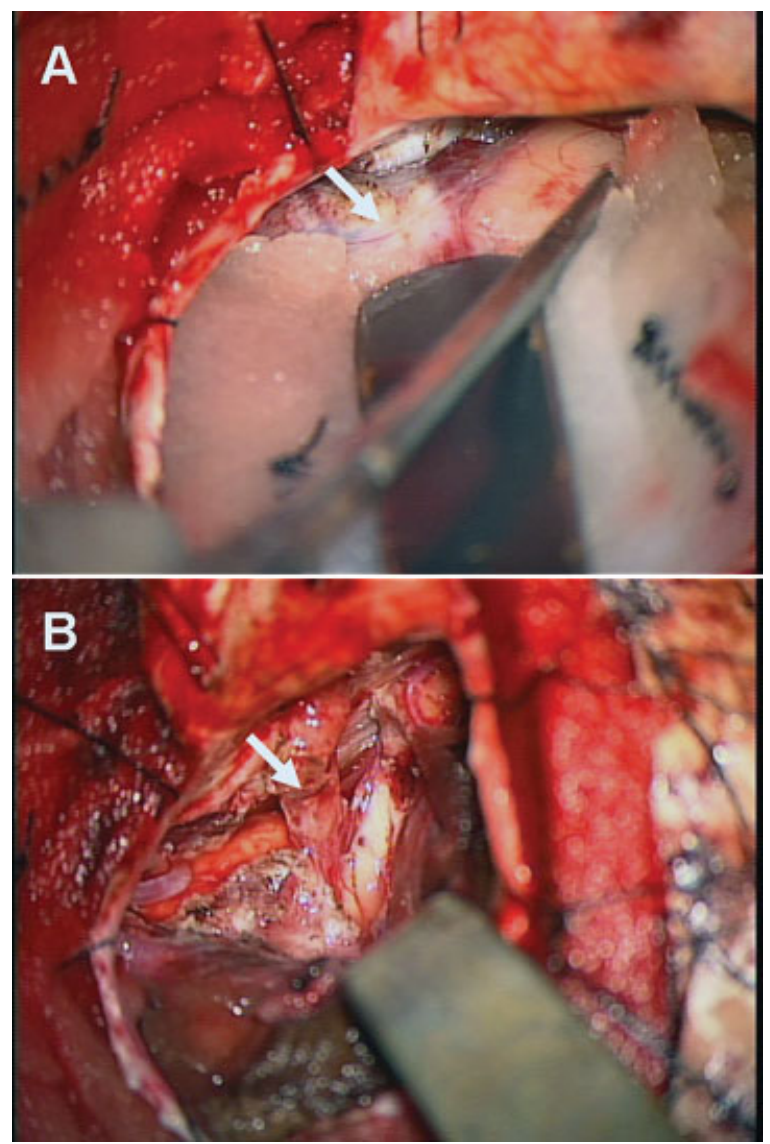

Fig. 3 Intraoperative view: an extra-arachnoid tumor involving the ninth and tenth cranial nerves. (A) Arachnoid membrane over the tumor (arrow). (B) No clear plane was observed between the tumor and nerves (arrow), suggesting an infiltrating lesion. sion of the suboccipital and parapharyngeal lesion following $40-G y$ irradiation, but the lower cranial nerve palsy did not recover. He developed diarrhea 1 month after the radiation therapy. Whole-body CT and gallium-67 scintigraphy showed multiple metastatic lesions in the mediastinum, liver, spleen, and gastrointestinal tract. The patient and his family refused further treatment, and he died from aspiration pneumonia in January 2010, 20 months after the first symptoms and 3 months after the histopathologic diagnosis of diffuse large B-cell lymphoma. The postmortem pathologic examination showed multiple metastatic lesions of the mediastinum, liver, spleen, lung, kidney, and gastrointestinal tract, as well as lymph nodes throughout the body. We did not observe a recurrence of the primary intracranial and parapharyngeal lesion across the jugular foramen. Lymphoma infiltration in the brain and meninges was not observed.

\section{Discussion}

Neurolymphomatosis is a rare type of lymphoma characterized by a direct invasion of the peripheral nervous system by the lymphoma, which is usually B-cell non-Hodgkin lymphoma in most cases. ${ }^{1,2,8}$ The diagnostic criteria for primary neurolymphomatosis is the first manifestation of the hematologic malignancy and proof of lymphoma cell infiltration in the affected neural structure is confirmed by biopsy or autopsy. ${ }^{2}$ It is important to distinguish neurolymphomatosis of the cranial nerves from PCNSL. Cranial nerve invasion by PCNSL is usually accompanied by subarachnoid seeding, whereas neurolymphomatosis of the cranial nerves localizes outside the arachnoid investment of the cranial nerves. ${ }^{9}$ Based on these definitions, the present case is compatible with primary neurolymphomatosis of the lower cranial nerves.

DWI and serum soluble interleukin-2 receptor (sIL-2R) have been reported to be of diagnostic and prognostic value in patients with malignant lymphomas. ${ }^{10,11}$ Early investigation demonstrated that densely packed tumor cells with a high nuclear-to-cytoplasmic ratio could be detected by DWI. ${ }^{12-15}$ Although the DWI characteristics of neurolymphomatosis have not been reported, it has been reported that DWI 


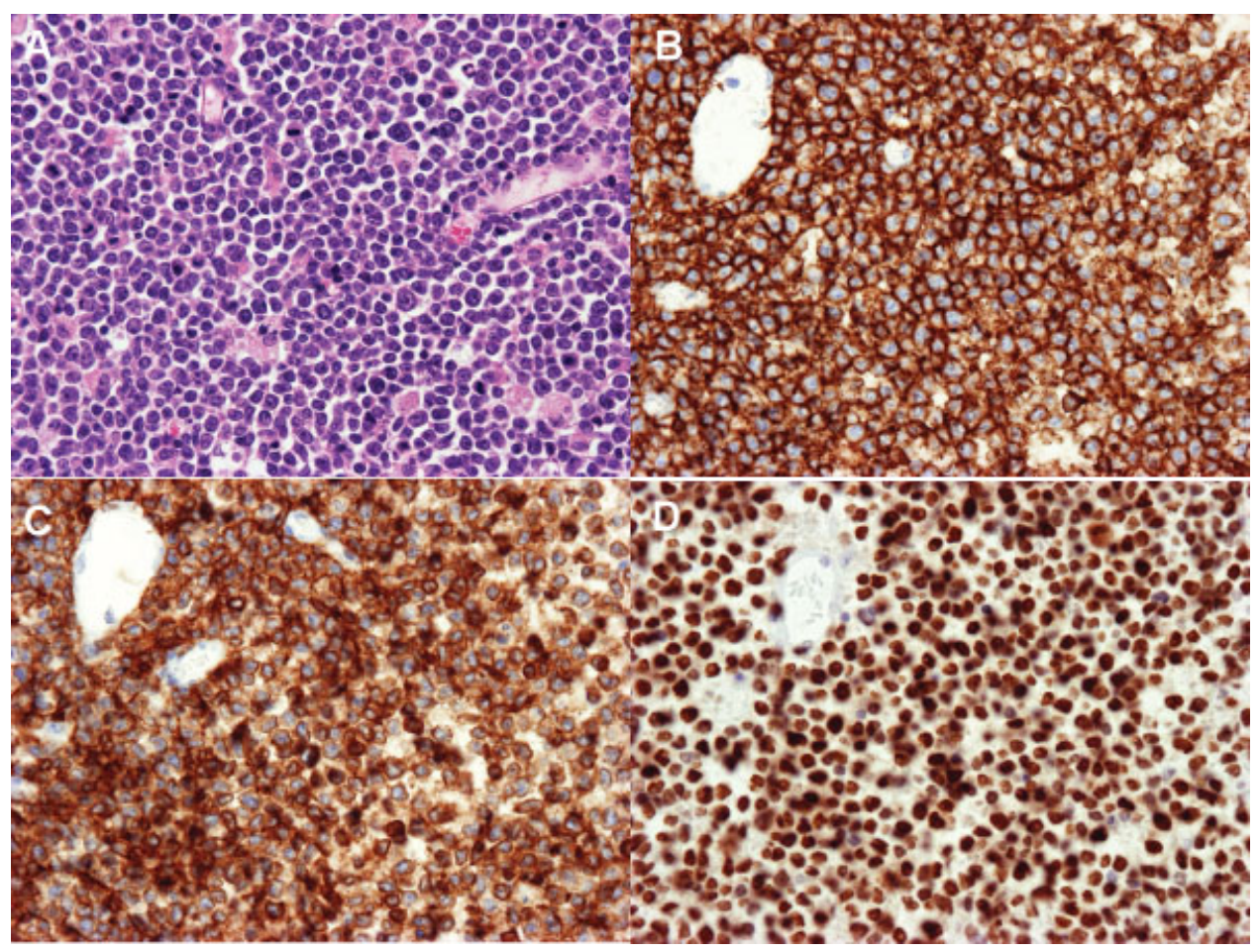

Fig. 4 (A) Hematoxylin-eosin staining and (B) immunohistochemical staining for CD20, (C) CD79, and (D) MUM1. Magnification $\times 400$.

correlates with histopathologic assessment of tumor cellular density in PCNSL. ${ }^{14}$ It was reported that DWI is predictive of clinical outcome in PCNSL. ${ }^{10}$ sIL-2R is the soluble form of the interleukin-2 receptor. The interleukin-2 receptor is expressed on the cell membrane of lymphocytes and plays an important role in their activation and proliferation. ${ }^{16}$ It has been reported that a high serum sIL-2R level is an indicator of poor prognosis in diffuse large B-cell lymphomas. ${ }^{11}$ In the present case, the tumor was isointense to hyperintense on DWI and the serum sIL-2R level was higher than normal. These findings were consistent with malignant lymphoma.

Because of the rarity of the disease, no standard treatment for neurolymphomatosis has been established. ${ }^{2}$ Treatments in the previously reported cases varied from systemic chemotherapy to intra-cerebrospinal fluid chemotherapy to radiotherapy. Systemic chemotherapy included high-dose methotrexate, high-dose cytarabine, and other combination chemotherapies including rituximab-Cytoxan, hydroxyrubicin, Oncovin, and prednisone. ${ }^{1,2}$ Rituximab is a chimeric murine monoclonal antibody against the $\mathrm{CD} 20$ antigen on B-cell lymphoma cells that has limited efficacy against PCNSL because of poor penetration into the cerebrospinal fluid. ${ }^{8}$ However, because neurolymphomatosis is located outside the central nervous system, rituximab might have potential therapeutic efficacy. ${ }^{8}$ Radiation has limited effectiveness in neurolymphomatosis treatment. ${ }^{2}$ Extended radiation fields are poorly tolerated in most patients, but limited field radiotherapy is very effective in relieving unremitting neuropathic pain attributed to a particular nerve, plexus, or nerve root. ${ }^{2}$ The median overall survival for primary neurolymphomatosis is reported to be 10 months, with $46 \%$ of patients surviving to 12 months and $24 \%$ surviving to 36 months. $^{2}$
In the present case, the patient was treated by tumor resection and focal radiation therapy without chemotherapy. Focal irradiation resulted in complete remission of the intracranial and parapharyngeal lesions and relieved the intolerable occipital and neck pain. Systemic chemotherapy with intravenous high-dose methotrexate might be the most effective initial treatment, ${ }^{2}$ but it could not be used in the present case because of the generally poor condition of the patient. As a result, secondary systemic lymphoma occurred soon after the radiation therapy. Early diagnosis of primary neurolymphomatosis is necessary for an aggressive multimodality therapy.

\section{Conclusions}

Diagnosing primary neurolymphomatosis of the cranial nerves is challenging, but meticulous use of imaging techniques such as MR imaging with DWI and FDG PET-CT, as well as evaluating serum sIL-2R levels, can help detect early stages of the disease. If neurolymphomatosis of the cranial nerves is suspected, a rapid pathologic diagnosis and early aggressive multimodality therapies are essential for ensuring prolonged patient survival.

\section{References}

1 Baehring JM, Damek D, Martin EC, Betensky RA, Hochberg FH. Neurolymphomatosis. Neuro Oncol 2003;5(2):104-115

2 Grisariu S, Avni B, Batchelor TT, et al; International Primary CNS Lymphoma Collaborative Group. Neurolymphomatosis: an International Primary CNS Lymphoma Collaborative Group report. Blood 2010;115(24):5005-5011 
$3 \mathrm{Kim}$ JH, Jang JH, Koh SB. A case of neurolymphomatosis involving cranial nerves: MRI and fusion PET-CT findings. J Neurooncol 2006;80(2):209-210

4 Santos E, Scolding NJ. Neurolymphomatosis mimicking neurosarcoidosis: a case report. J Med Case Reports 2010;4:5

5 Hung MH, Gau JP. Relapsed testicular lymphoma presenting with cranial nerve neurolymphomatosis. Neurology 2011;76(16):1441

6 Iplikcioglu AC, Dinc C, Bikmaz K, Ozcan D. Primary lymphoma of the trigeminal nerve. Br J Neurosurg 2006;20(2):103-105

7 Levin N, Soffer D, Grissaru S, Aizikovich N, Gomori JM, Siegal T. Primary T-cell CNS lymphoma presenting with leptomeningeal spread and neurolymphomatosis. J Neurooncol 2008;90(1):77-83

8 Gan HK, Azad A, Cher L, Mitchell PL. Neurolymphomatosis: diagnosis, management, and outcomes in patients treated with rituximab. Neuro Oncol 2010;12(2):212-215

9 Epelbaum R, Haim N, Ben-Shahar M, Ben-Arie Y, Feinsod M, Cohen Y. Non-Hodgkin's lymphoma presenting with spinal epidural involvement. Cancer 1986;58(9):2120-2124

10 Barajas RF Jr, Rubenstein JL, Chang JS, Hwang J, Cha S. Diffusionweighted MR imaging derived apparent diffusion coefficient is predictive of clinical outcome in primary central nervous system lymphoma. AJNR Am J Neuroradiol 2010;31(1):60-66

11 Ennishi D, Yokoyama M, Terui Y, et al. Soluble interleukin-2 receptor retains prognostic value in patients with diffuse large B-cell lymphoma receiving rituximab plus $\mathrm{CHOP}$ (RCHOP) therapy. Ann Oncol 2009;20(3):526-533

12 Le Bihan D, Turner R, Douek P, Patronas N. Diffusion MR imaging: clinical applications. AJR Am J Roentgenol 1992;159(3): 591-599

13 Rowley HA, Grant PE, Roberts TP. Diffusion MR imaging. Theory and applications. Neuroimaging Clin N Am 1999;9(2):343-361

14 Guo AC, Cummings TJ, Dash RC, Provenzale JM. Lymphomas and high-grade astrocytomas: comparison of water diffusibility and histologic characteristics. Radiology 2002;224(1):177-183

15 Stadnik TW, Chaskis C, Michotte A, et al. Diffusion-weighted MR imaging of intracerebral masses: comparison with conventional MR imaging and histologic findings. AJNR Am J Neuroradiol 2001; 22(5):969-976

16 Takeshita T, Asao H, Ohtani K, et al. Cloning of the gamma chain of the human IL-2 receptor. Science 1992;257(5068):379-382 